\title{
Universal Heliophysical Processes
}

\author{
Nat Gopalswamy
}

\begin{abstract}
The physical processes in the heliospace are a direct consequence of the influenced by Sun's mass and electromagnetic emissions. There has been enormous progress in studying these processes since the dawn of the space age half a century ago. The heliospace serves as a great laboratory to study numerous physical processes, using the vast array of ground and space-based measurements of various physical quantities. The observational capabilities collectively form the Great Observatory to make scientific investigations not envisioned by individual instrument teams. The International Heliophysical Year (IHY) program has been promoting scientific investigations on the universality of physical processes such as shocks, particle acceleration, dynamo, magnetic reconnection, magnetic flux ropes, plasmaneutral matter interactions, turbulence, and so on. This paper highlights scientific deliberations on these and related topics that took place during the IAGA session on "Universal Heliophysical Processes" in Sopron, Hungary. The session featured several invited and contributed papers that focused on observations, theory and modeling of the universal heliophysical processes.
\end{abstract}

\section{Introduction}

The Sun strongly influences a region of space around it to a distance of about 100 AU by virtue of its mass and electromagnetic emissions. In-situ and remote-sensing observations combined with theory and modeling efforts have helped accumulate a wealth of knowledge on the heliospace. The magnetized plasma, energetic particles, and the electromagnetic radiation from the Sun interact with the neutral and charged fluid envelopes that surround the planets and their moons. Neutral material from the interstellar medium and charged galactic origin also enter the heliospace re-

\footnotetext{
Nat Gopalswamy

NASA Goddard Space Flight Center, Greenbelt, MD 20771, USA e-mail: nat.gopalswamy@nasa.gov
} 
sulting in additional physical processes. The interaction between the solar wind and the interstellar neutral matter results in pickup ions. The magnetic enhancements in the solar wind caused by solar eruptions deflect the galactic cosmic rays, reducing their severity in impacting Earth. The International Geophysical Year (IGY) during 1957-1958 provided a major impetus to the development of space science. During 2007-2009, the International Heliophysical Year (IHY 2007) was conducted to take stock of the enormous achievements that the world scientific community has accomplished since 1957 (see Davila et al, 2010, for an overview of the IHY program and its continuation as the International Space Weather Initiative (ISWI)). The IAGA session on "Universal Heliophysical Processes" in Sopron, Hungary provided a forum to discuss these achievements. Invited and contributed papers addressed issues on the variability of the sun from its interior to its atmosphere and in the extended heliospace.

\section{Solar Interior, Dynamo, and the Solar Cycle}

The mass and electromagnetic emissions from the Sun are ultimately related to the generation of energy in the solar core and its transport by radiative and convective processes in the solar interior. The energy generation in the solar core is primarily due to the thermonuclear reactions that convert hydrogen to helium. Such a conversion is consistent with the stellar structure inferred from helioseismology, a field that developed rapidly with its origin in the discovery of the 5-minute solar oscillations (Leighton and Brophy, 1961). The interior structure and dynamics of the Sun has been established to a very high degree of accuracy by studying the acoustic waves trapped inside the Sun. The solution to the solar neutrino problem (Ahmad et al, 2001) also confirmed the standard solar model established by helioseismology many years earlier. Couvidat (2010) summarized the current developments in the global and local helioseismology branches. The local helioseismology is concerned with sunspot regions, where one observes intense magnetic fields produced at the base of the convection zone and transported to the surface. The sunspot regions are the sources of the most violent eruptions whose consequences can be felt throughout the heliosphere.

\subsection{Solar Activity Cycles}

The generation and maintenance of solar magnetism by the differential rotation of the Sun and the turbulent convection in its outer layers is central to most of the energetic phenomena that take place in the solar atmosphere. The dynamo mechanism involves the conversion of the poloidal field into toroidal field and vice versa resulting in the 11-year sunspot cycle and the 22-year magnetic cycle (see e.g., Charbonneau, 2005, for a review). The sunspot regions are the source of coronal mass 
ejections (CMEs) and flares that lead to hazardous space weather. The largest number of sunspots appears on the Sun during the solar maximum, when likelihood of energetic eruptions is very high. Prediction of the sunspot number is therefore of great practical importance and provides a metric of our understanding of solar magnetism. Petrovay (2010) reviewed empirical and theoretical regularities and patterns underlying the sunspot cycle and discussed recent model-based prediction attempts for solar cycle 24. Petrovay also discusses the possible mechanisms that explain the amplitude of the solar activity modulated over a longer time-scale ( $\sim 90 \mathrm{yr})$ resulting in epochs of suppressed solar activity (grand minima). There is widespread interest in grand minima after Eddy (1976) discovered the Maunder minimum and its implications for Earth's climate.

There have been attempts, both based on empirical and dynamo models, to predict solar cycles. The sunspot number predictions for cycle 23 occupy a huge range above and below the observations for cycle 23 (see e.g., Brajša et al, 2009). Using a combined method, Brajša et al (2009) predicted the strength and the epoch of cycle 24: the sunspot number will be in the low 80s, occurring around the year 2012. Verbanac et al (2009) presented the details of this prediction method: (1) the calculation of the asymmetry of the duration of the ascending and descending solar cycle parts, (2) the correlation of the relative sunspot numbers in and around solar activity minima and the following activity maxima and (3) the method of the autoregressive moving average model (ARMA) applied to the relative sunspot number data measured up to now. They utilized various data sets that comprise of yearly, corrected yearly, monthly and smoothed monthly relative sunspot number values.

\subsection{Solar Dynamo and Grand Minima}

Recognizing solar activity cycles from various proxy data has greatly enhanced our understanding of solar variability. Demetrescu et al (2009) used annual means of measured and reconstructed solar, heliospheric, and magnetospheric parameters to infer solar activity signatures at the Hale magnetic (MC) and Gleissberg cycle (GC) timescales. They reconstructed available open solar flux, modulation strength, cosmic ray flux, and total solar irradiance data back to 1700; solar wind parameters (speed and density) and the magnitude of the heliospheric magnetic field at $1 \mathrm{AU}$ were reconstructed back to 1870; time series of geomagnetic activity indices (aa, IDV, IHV), going back to 1870 , were also considered. Simple filtering procedures (successive 11-, 22-, and 88-year running averages and differences between them) and scaling by the standard deviation from the average value for the common interval covered by the data show that the long-discussed variation in the 20th century (a pronounced increase since 1900 , followed by a depression in the 1960s and a new, slower, increase) seen in the 11-year averages of the analyzed parameters, is a result of the superposition in data of solar activity signatures MC and GC timescales (see Fig. 1). This leads to the conclusion that the MC and GC signals are quite similar in all studied parameters, pointing to a common pacing source, the solar dynamo. 


\section{Solar Eruptions and their Interplanetary Consequences}

It is possible to recognize distinct activity phenomena associated with the toroidal and poloidal fields of the Sun. Flares, CMEs, and irradiance variations are connected to the toroidal fields, while the high-speed solar wind from solar poles is linked to the poloidal field. Both sets of phenomena have important consequences on Earth. Thus the variations in the action of the solar dynamo can result in varying influences on the geoeffectiveness and terrestrial climate (Georgieva, 2009). Flares, high-speed solar wind, and CMEs produce space weather effects, while irradiance variation produces climate effects. CMEs impact on Earth's magnetosphere and result in intense geomagnetic storms, while high-speed solar wind can produce moderate and weak storms. Flares produce short-term ionospheric disturbances that can

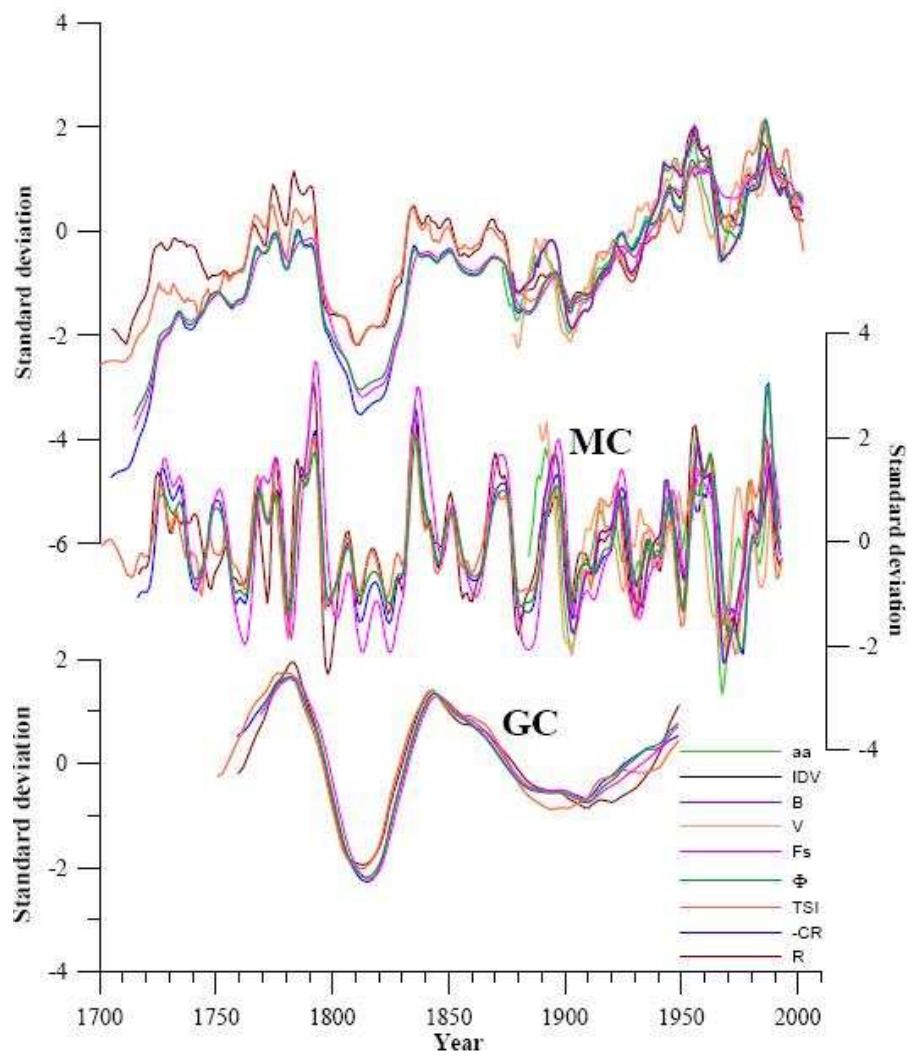

Fig. 1 11-year averages (top), the Hale magnetic cycle (MC) signal (middle) and the Gleissberg (GC ) signal (bottom) in various data: aa - geomagnetic activity index , IDV - geomagnetic interdiurnal variability index, B - magnitude of the heliospheric magnetic field; V - solar wind speed; Fs - open solar flux; $\Phi$ - heliospheric modulation strength; TSI - total solar irradiance; CR - cosmic ray flux (reconstructed count rate of the standard neutron monitor); $\mathrm{R}$ - sunspot number. 
affect radio communication and navigations and accelerate solar energetic particles (SEPs) of the impulsive type. CMEs on the other hand drive fast mode MHD shocks that accelerate gradual SEPs, which are significant because of they are potentially hazardous to space-based technological systems and humans in space.

CMEs originate from closed magnetic regions that possess free magnetic energy. Active regions produce the most energetic CMEs because of the large free energy that can be stored in them. One of the important indicators of free energy is the active region helicity. The helicity generation is thought to be closely connected with the toroidal and poloidal fields (Longcope et al, 1998; Choudhuri et al, 2004). Based on the estimates of magnetic helicity in interplanetary flux ropes, Sung et al (2009) showed that the CME kinetic energy and the flux rope helicity are closely related. Thus, the active region helicity and the flux rope structure play a significant role in understanding the generation and interplanetary consequences of CMEs.

There is plenty of observational evidence for flux rope structure in CMEs and their interplanetary counterparts (ICMEs). The flux rope structure was inferred from the solar wind magnetic field data (see e.g., Burlaga et al, 1981). The coronagraphic observations from the Solar and Heliospheric Observatory (SOHO) reveal flux rope structure (see e.g., Chen et al, 1997). Linton (2010) compared the CME flux rope structure with the plasmoids observed in the Earth's magnetotail, though the two structures are of vastly different spatial scale. With this comparison, Linton concluded that flux rope formation is a universal space physics phenomenon and that the physical mechanisms responsible for flux rope formation occur over a wide range of plasma conditions wherever current sheets exist. The current sheets in the solar atmosphere are of particular interest it is supposed to result in the CME flux ropes. Poletto (2010) reviewed the observational evidence of current sheets throughout the solar atmosphere.

One of the important consequences of a flux rope structure is that we can predict which portion of the flux rope is likely to interact with Earth's magnetosphere. For example, if the flux rope axis is in the ecliptic plane, then the leading field is pointed to the north or south, while the trailing field has the opposite sense. South-pointing field is necessary for reconnection with the magnetospheric field, which points to the north. Another advantage is the possibility that the flux rope axis is parallel to the neutral line in the solar active region (or the axis of the filament overlying the neutral line). Observations indicate that there is significant deviation between the flux rope axis and the active region neutral line, suggesting that the field orientations rotate by up to 160 degrees with respect to the magnetic orientation of the CME source region on the Sun. The cause of this rotation it is not well understood. Török et al (2009) reported on a systematic study of CME rotation mechanisms, based on numerical MHD simulations of a flux rope CME model. They focused on two mechanisms: (i) the conversion of flux-rope twist into writhe and (ii) the interaction of the fluxrope current with the component of the ambient coronal magnetic field along the flux rope. By varying the initial flux rope twist and the shear angle of the ambient magnetic field, they found a continuous range of flux rope rotations between $\sim 20$ and $\sim 140$ degrees in the simulations. Strong rotations of more than $\sim 100$ degrees require both high flux rope twist (i.e., the occurrence of the helical kink instability) 
and the presence of a significant shear of the ambient field with respect to the initial flux rope orientation.

Occasionally, linking the active region helicity to that of the interplanetary flux rope originating from the active region may not be straightforward. Chandra et al (2010) discussed a CME from NOAA AR 10501 that was associated with a magnetic cloud (MC) of positive magnetic helicity on 20 November, 2003. However, the active region had a negative helicity, opposite to that of the MC. They reconciled the disparity using the observation that a smaller region within the active region had emerging flux with a positive helicity, which might explain the positive helicity in the MC.

\section{CME-driven Shocks and Related Phenomena}

The heliospace plasma is an ideal laboratory for collisionless shock physics, because it supports a variety of shocks, ranging from mini-bow shocks ahead of the lunar magnetic anomaly to large scale shocks in front of CMEs (Terasawa, 2010). The dissipation processes at collisionless shocks naturally result in the production of nonthermal particles. CME-driven shocks are of particular interest because they accelerate electrons and ions throughout the heliospace. Shock waves near the Sun produce type II radio bursts and release SEPs (see Valtonen, 2010). Type II bursts represent one of the several different radio emission processes that occur in the heliospace (Messerotti, 2010). When the shocks impact the magnetosphere, they produce the storm sudden commencements, which are signatures of magnetospheric compression.

\subsection{EUV Wave Transients}

The CME-driven shocks also manifest as EUV wave transients, which are thought to be bow waves enveloping CMEs. Veronig (2010) reviewed the current status of large-scale EUV waves (also known as "EIT waves" and "coronal Moreton waves") in the solar corona that are launched in association with solar flares and CMEs. In particular she discussed the physical nature of the wave: blast wave, driven-wave, or non-waves. Based on the wave kinematics and dynamics derived from high-cadence observations by the EUVI instruments onboard the twin STEREO spacecraft, she concluded that the coronal wave reveals deceleration, indicative of a freely propagating MHD wave after an initial driving by the expanding flanks of the associated CME. When EIT waves are associated with type II radio bursts, one infers that the EIT wave is in fact a fast mode shock. 


\subsection{Radio-loud and Radio-quiet Shocks}

The fact that shocks are important source of energetic particles in the heliosphere can be inferred in a number of ways. Type II radio bursts are the earliest signature of particle acceleration by CME-driven shocks. Type II bursts begin when the CMEs are typically at a heliocentric distance of $1.5 \mathrm{Rs}$ (Gopalswamy et al, 2009b) indicating that electrons accelerated to an energy of up to $10 \mathrm{keV}$ escape from the CME-driven shock front. Type II bursts also serve as an indicator of particle acceleration by shocks throughput the inner heliosphere. SEPs are released typically at larger heliocentric distances ( 5-7 Rs), where a combination of circumstances favor SEP acceleration and release (CMEs reaching peak speeds and Alfven speed starts declining). This spatial domain overlaps with the fields of view of white-light coronagraphs, so shock signatures can be discerned in the coronagraphic images as the diffuse feature ahead of the bright structures. Gopalswamy et al (2009a) described the diffuse feature as the compressed sheath ahead of the flux rope in the 2005 January 15 CME (see Fig. 2). The extent of the diffuse feature differed substantially when shocks with (radio loud) and without type II bursts (radio quiet) were considered. They investigated the widths of the flux rope and the surrounding disturbance for 13 radio-quiet and 47 radio-loud interplanetary shocks associated with limb CMEs. The sky-plane widths of the surrounding disturbance were much larger for the case of radio-loud shocks (284 degrees compared to 197 degrees for the radio-quiet shocks).

\subsection{Extended Shocks and SEPs}

The large extent of the shock surrounding energetic CMEs has important consequences in the heliosphere. Malandraki et al (2009) reported energetic particle ob-
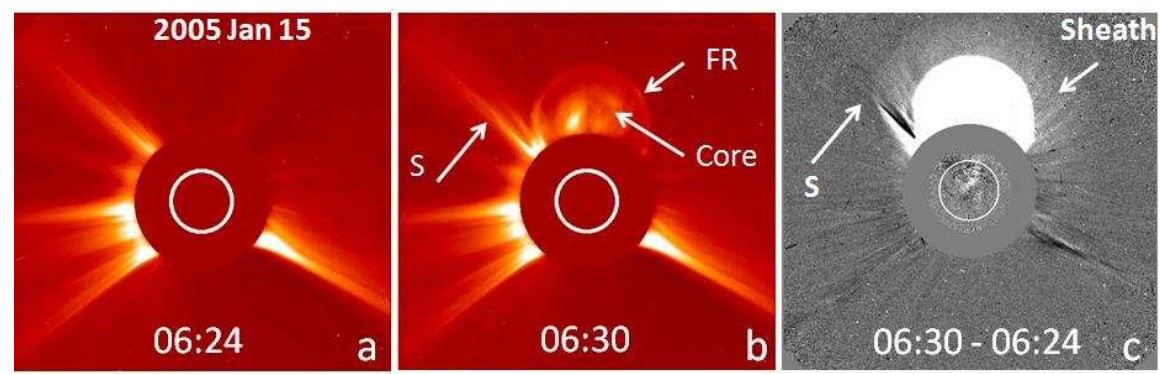

Fig. 2 A flux rope CME (2005 January 15) observed by SOHO/LASCO with the surrounding shock disturbance. (left) pre-CME corona at 6:24 UT, (middle) the CME with flux rope (FR) and prominence core and a kink (S) in the streamer outside the flux rope, and (right) The difference between the images at 06:30 and 06:24 showing the diffuse material (sheath) surrounding the flux rope. 
servations by Ulysses during the period of isolated but intense solar activity from active region 10930 in December 2006. The heliocentric distance of Ulysses was 2.8 $\mathrm{AU}$, located at $72^{\circ}$ southern heliographic latitude immersed in the high-speed solar wind from the southern polar coronal hole. The particle event was associated with an X9.0 flare from S07E79 on December 5, being the largest among a set of 4 Xclass events. Three forward shocks from these eruptions were observed at Ulysses, even though Ulysses was located at W120. The shocks were not followed by discernible ejecta suggesting that the shocks were much more extended than the ejecta. The onset and decay profiles of the event for the 8-19 MeV proton channel were relatively smooth. The event had a 'clean' onset, occurring in a period nearly devoid of solar wind structures and with relatively low pre-event intensities (see Fig. 3). The pitch-angle distributions of energetic particles were near-isotropic. The particle flow directions were along the field and there was no evidence for any net flow across the field lines, so there was little cross-field diffusion close to the observer. The magnetic field in the fast solar wind is much more turbulent, so particle propagation should be more difficult leading to significant scattering. The rise-time of the SEP event at STEREO-B was found to be faster than at Ulysses (although this corresponds to an eastern poorly connected event as viewed from the Earth), suggesting a more diffusive transport to high latitudes than to the near-Earth STEREO-B. The relatively simple structure of the heliosphere in December 2006 when Ulysses was in the southern polar wind seem to exclude the possibility that magnetic field lines originating at low-latitudes reached Ulysses. The energetic particles observed as large SEP events over the south pole of the Sun were released when the propagating coronal waves reached high latitude magnetic field lines connected to Ulysses. Based on the observations available, however, cross-field diffusion closer to the Sun cannot be definitely excluded.

\subsection{Geospace Consequences of Solar Eruptions}

CMEs have important consequences in geospace by direct impact (geomagnetic storms) and via SEPs, which can be trapped in Earth's radiation belt over long periods of time. SEPs can also penetrate Earth's atmosphere at high latitudes leading to ozone depletion. The properties of magnetosphere can also be modified during geomagnetic storms. Kudela and Lazutin (2010) reviewed studies on the cosmic ray anisotropy observed at neutron monitors and muon telescopes just before the onset of some geomagnetic storms. They also discussed changes of geomagnetic cut-off, structure of the cosmic ray transmissivity function and the asymptotic directions for various geomagnetic field models during strong geomagnetic storms. They reported preliminary results of a study on the solar proton capture into radiation belts using coronas data and explored the use of different geomagnetic field models that can fit the observed trapped particle profiles in different local time sectors. Furthermore, they pointed out that measurements of energetic neutral emissions (gammas and 
neutrons) near the Earth or on the ground can serve as indicators of acceleration processes on solar surface.

The first interplanetary structure that impacts Earth's magnetosphere is the shock ahead of the ICME, which is recognized in the ground-based magnetometer data as a sudden increase in the horizontal component of Earth's magnetic field known as the geomagnetic sudden commencement (SC). The preliminary impulse (PI) of SCs appears as a negative impulse (PRI) at the afternoon high latitude and dayside geomagnetic equator, and a positive impulse (PPI) at morning high latitude and nightside geomagnetic equator. The temporal and spatial variations of the PI are explained by means of a magnetosphere-ionosphere current system, composed of the ionospheric Hall and Pedersen currents at high latitudes and the Pedersen current amplified by the Cowling effect at the equator. The ionospheric currents are driven by the dusk-to-dawn electric field impressed from the magnetosphere. The electric field then propagates to low latitude, driving westward/eastward currents in the day/nightside equatorial ionosphere. Although the observations indicate instantaneous transmission of the electric field to the equator, a complete confirmation required high time resolution and better data coverage. Kikuchi et al (2010) analyzed PI events using 1-sec sampled magnetometer data recorded at high-equatorial latitudes on both day- and nightside. They found that the PI to start simultaneously with the temporal resolution of $1 \mathrm{sec}$ at all latitudes and local times. The D-component deflections at mid latitudes were consistent with the Pedersen currents connecting the field-aligned currents with the equatorial PI currents. The instantaneous devel-
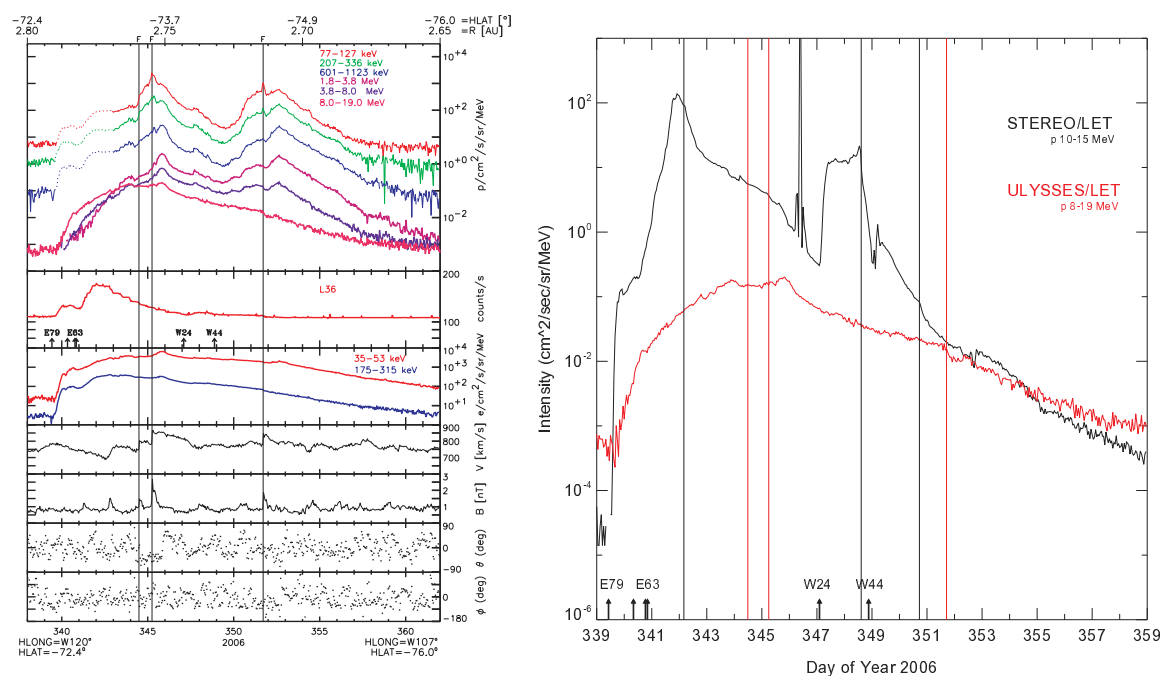

Fig. 3 (left) ion and electron intensity profiles and solar wind plasma and magnetic field data from Ulysses. (right) hourly averages of proton intensities at similar energy intervals measured in and out of the ecliptic plane by the COSPIN/LET and STEREO/LET experiments onboard Ulysses (red trace) and STEREO-B (black trace) during the December 2006 period. courtesy: O.E. Malandraki (IAA/NOA). 
opment of the PI currents is consistent with their scenario based on the TM0 mode wave in the Earth-ionosphere waveguide.

Nanan et al (2010) studied the changes in the Earth's ionosphere due to the passage of the CMEs of 07-11 November 2004. The observed changes include (1) the direct response of the high latitude ionosphere, (2) the development of a rare super double geomagnetic storm, (3) the development of strong daytime eastward prompt penetration electric field (PPEF) events in the longitudes that were in daytime sectors during the main phases of both super storms, (4) the strong F3 layer with large density depletions around the equator in the longitudes of the PPEF events, and (5) the large positive/negative ionospheric storms at low-mid latitudes. Using the physics based model SUPIM, they evaluated the relative importance of diffusion, daytime eastward PPEF and neutral wind on the equatorial plasma fountain and positive ionospheric storms. The plasma fountain was found to rapidly develop into a super fountain and the equatorial ionization anomaly (EIA) crests shifted to higher than normal latitudes during the PPEF event both in the presence and absence of neutral winds. However, the super fountain became stronger with less poleward turning of the plasma flux vectors and the EIA crests became stronger than normal in the presence of an equatorward wind. The equatorward wind reduced (or stopped) the downward velocity component due to diffusion and raised the ionosphere to high altitudes of reduced chemical loss. These mechanical effects of the equatorward wind accumulated the plasma brought by the super fountain, and hence strengthened the EIA crests and produced positive ionospheric storms; the wind need not be a stormtime wind, although stronger wind could lead to stronger ionospheric storms.

\section{Solar Wind Processes}

Solar wind represents another important mass emission process from the Sun, which is rather steady in contrast to that during CMEs. The large number of observations and theoretical studies of the solar wind since its original prediction by Parker (1958) have established the basic picture that the wind is driven by heating of the solar corona. Lie-Svendsen (2010) emphasized the need to treat the corona and wind as one tightly coupled system. He also noted that the solar mass loss is controlled by the amount of coronal heating and by the energy flow between the chromosphere and corona. He also highlighted the following key results from SOHO/UVCS observations: (i) protons and heavy ions are much hotter than electrons, (ii) the wind is rapidly accelerated within a few solar radii, and (iii) the ion temperature perpendicular to the magnetic field is much higher than the parallel temperature. From these observations he concluded that the solar wind is driven mainly by proton heating close to the Sun, perhaps through ion cyclotron waves. 


\subsection{Evolution of Solar Wind Properties}

The twin spacecraft of the STEREO mission have helped understand the temporal evolution of the solar wind plasma properties using the electron and ion measurements. Opitz et al (2009a) compared the proton bulk velocity measurements made by the PLASTIC instruments on STEREO A and B after adjusting for the theoretical time lag. They obtained the correlation coefficients for the proton bulk velocity as a function of the time difference between two ejections of the plasma parcel from the solar source as 0.95 and 0.85 for time lags of 0.5 days and 2 days, respectively (Fig. 4a). They performed a similar correlation analysis of the electron core densities measured by the two STEREO/IMPACT instruments. The correlation coefficients were 0.80 and 0.65 for time lags of 0.5 days and 2 days, respectively (Fig. 4b). The correlation was lower for the electron density than for the proton bulk velocity, though for both parameters the solar wind can be considered as persistent over more than 2 days. Opitz et al (2009b) extrapolated the solar wind bulk velocity measurements for different in-ecliptic heliospheric positions, which were validated using on-site measurements at Venus (VEX), Earth (SOHO) and Mars (MEX). They also showed that the solar wind is accelerated or decelerated at stream interfaces during its radial propagation.

\subsection{Solar Wind Turbulence}

The solar wind also offers a unique laboratory for studying turbulent plasma processes. Of particular interest is the turbulence at MHD scales because it strongly affects several aspects of the solar wind: generation and heating of the wind, particles acceleration, and cosmic-ray propagation. Alfvenic fluctuations in the solar wind are also linked to geomagnetic activity. Bavassano (2010) reviewed the solar
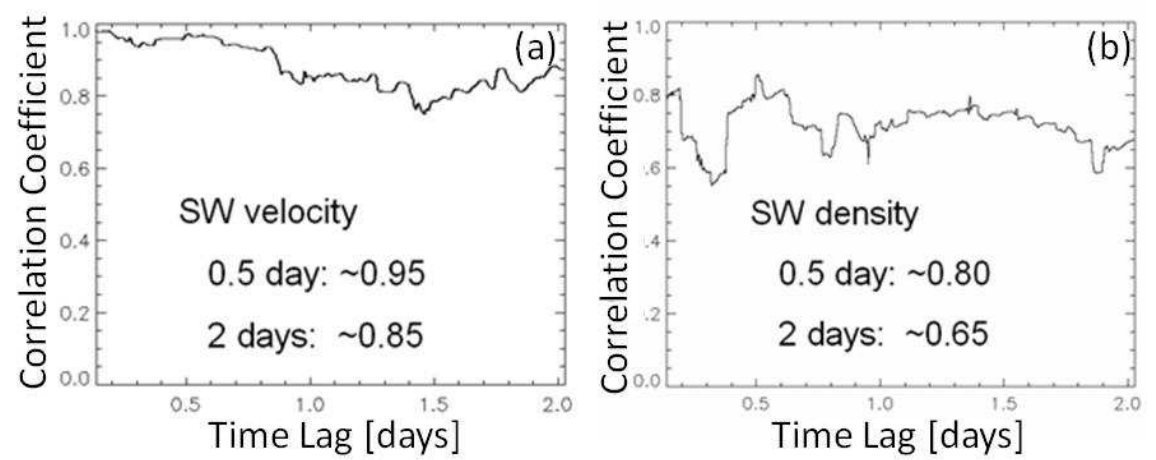

Fig. 4 Temporal evolution of the solar wind proton bulk velocity (a) and electron core density (b) obtained by correlating the STEREO A and B measurements. 
wind observations at different heliocentric distances and latitudes that have helped understand the mechanisms driving the solar wind turbulence. Gary (2009) presented their idea on the role of whistler fluctuations in the dissipation regime of solar wind turbulence. They proposed the whistler fluctuations as an alternative hypothesis to describe short-wavelength turbulence in the solar wind, because the kinetic Alfven waves may not contribute to a power law spectrum. Particle-in-cell simulation showed that the whistler cascade yields steep power-law power spectra consistent with observations. Vörös and Leubner (2009) reported on the occurrence of magnetic turbulence in the solar wind on the basis of a wavelet approach. They concluded that the solar wind turbulence is strongly localized and associated with large-scale structures, in particular shocks. The quiet inter-shock periods in between the localized turbulent fluctuations were found to be unrelated to turbulence or turbulent intermittency. They also pointed out that the misinterpretations caused by the stationarity assumption in the recent literature are significant and therefore suggested a revision of the basic idea of stationarity in solar wind turbulence.

\subsection{Reconnection exhaust in the solar wind}

The reconnection events, known to be central to many energetic phenomena in the heliospace, have recently been found to occur in the solar wind. Lavraud et al (2009) reported on the analysis of one such event in the heliospheric current sheet (HCS) crossed by WIND, ACE and STEREO (see Fig. 5). Although only WIND and ACE provided good ion flow data in accord with a reconnection exhaust, the magnetic field bifurcation typical of such exhausts was clearly observed at all spacecraft. Unambiguous strahl mixing within the exhaust was consistent with the sunward flow deflection observed at WIND and ACE and thus with the formation of closed magnetic field lines within the exhaust with both ends attached to the Sun. The strong dawnward flow deflection was consistent with the exhaust and X-line orientations obtained from minimum variance analysis (MVA) at each spacecraft so that the X-line was almost along the GSE Z-axis and duskward of all the spacecraft. The observation of strahl mixing in extended and intermittent layers outside the exhaust by STEREO A and B was consistent with the formation of electron separatrix layers surrounding the exhaust. This event also provided further evidence that balanced parallel and anti-parallel suprathermal electron fluxes are not a necessary condition for identification of closed field lines in the solar wind. In the present case the origin of the imbalance simply was the mixing of strahls of substantially different strengths from a different solar source each side of the HCS. The inferred exhaust orientations and distances of each spacecraft relative to the X-line show that the exhaust was likely non-planar, following the Parker spiral orientation. Finally, the separatrix layers and exhausts properties at each spacecraft suggest that the magnetic reconnection X-line location and/or reconnection rate were variable in both space and time at such large scales. 


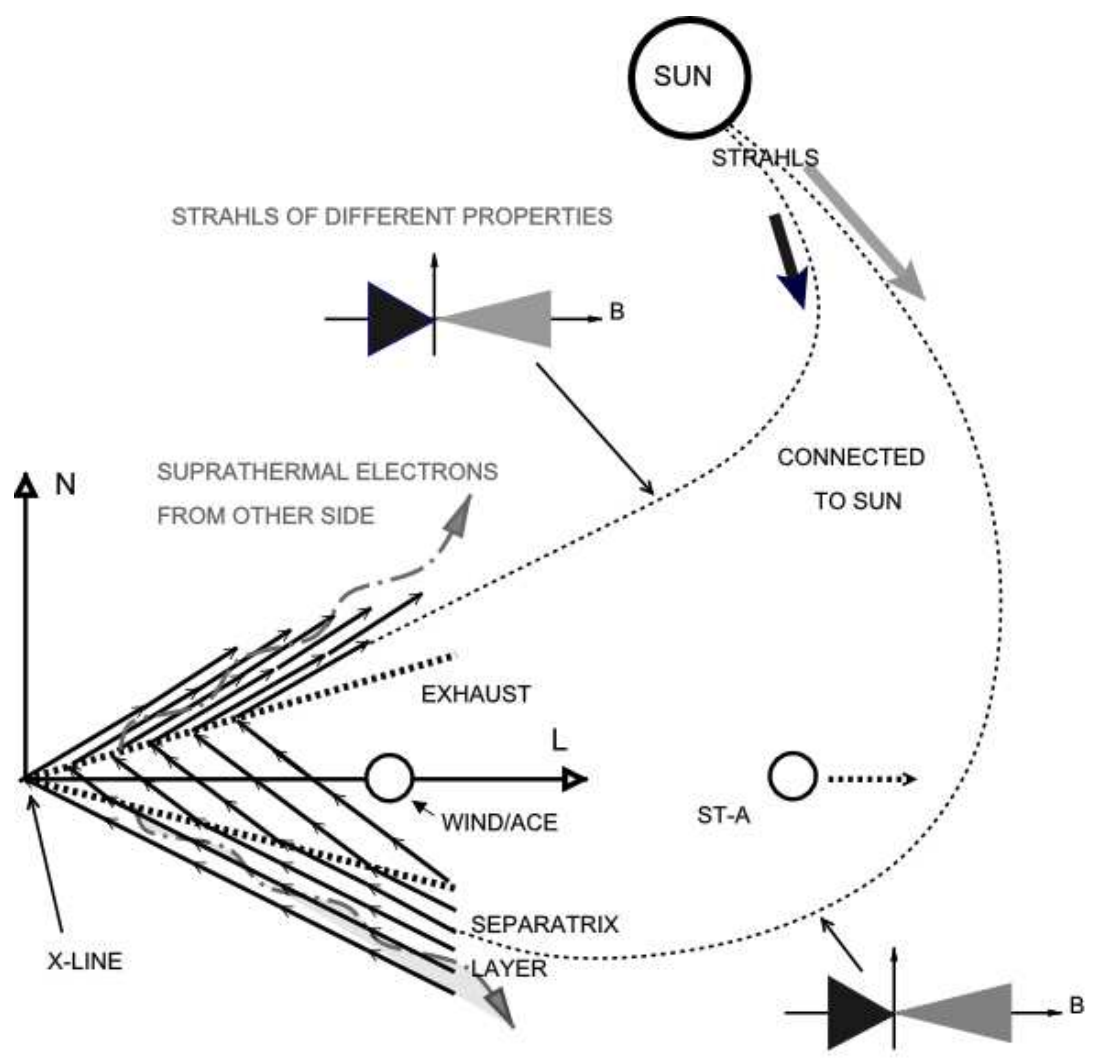

Fig. 5 (Lower left part) Idealized projection of asymmetric reconnection in the solar wind (in the $\mathrm{L}-\mathrm{N}$ plane formed by the directions of minimum (N) and maximum (L) magnetic field variance). The field line kinks at each exhaust boundary accelerate the plasmas they intercept. The exhaust boundaries are locally open, rotational-like discontinuities. Suprathermal electrons can flow from one side to the other (curvy dash-dotted lines), so as to form electron separatrix layers. (Right part of figure) Spacecraft such as Wind/ACE and STEREO-A, located on the sunward side of the reconnection site, are on newly closed field lines and thus observe the mixing of suprathermal electrons from the two sides of the reconnecting current sheet. The strahls from the two sides may be of different strengths (dark and light grey arrows at the Sun). From Lavraud et al (2009).

\subsection{Influence of the solar wind on energetic particles}

The solar wind plays also plays an important role in establishing the global characteristics of $\mathrm{MeV}$ particles in the inner heliosphere. Two aspects of this relation were reported by Kecskeméty et al (2009): the decay rate after solar energetic particle events and the energy spectrum during quiet solar activity. As convection and adiabatic deceleration play a dominant role in particle propagation at these energies, the characteristic time constant of exponential-form decays should be inversely proportional to solar wind speed resulting in an exponential time $(t)$ profile 
$J(t) \propto E^{-\gamma} \exp [-4 V(1+\gamma) t / 3 r]$ with $V$ denoting solar wind speed, $\gamma$ the slope of the energy $(E)$ spectrum, and $r$ the heliocentric distance. The characteristic decay time was found to be valid in more than 50\% of events in SOHO and Ulysses simultaneous time profiles; the $V$ and $r$ dependence was also confirmed. Based on IMP-8, $\mathrm{SOHO}$, and Ulysses data, the quiet-time $\mathrm{MeV}$ proton fluxes were found to correlate with solar activity, and in particular, with the solar wind speed. The comparison of particle fluxes and solar wind speed during the solar activity minima of 1976-77 and 1986-87 indicated that whereas fluxes of both quiet time low energy protons and GCR were lower in 1986-87, the corresponding solar wind speeds were higher. The two recent minima, 1996-97, and 2006-07 were also compared using SOHO data. The closest correlation was found between $4.5-20 \mathrm{MeV}$ proton fluxes and solar wind pressure (see Fig. 6, including quiet periods from the last two minima), the lowest proton fluxes appeared at lowest plasma pressures.

\subsection{Solar wind in the outer heliosphere}

The character of the solar wind undergoes a significant transformation in the outer heliosphere owing to the greater influence exerted by the interstellar medium. Using observations from multiple spacecraft distributed throughout the heliosphere and a multi-fluid MHD model, Wang and Richardson (2010) traced the propagation of

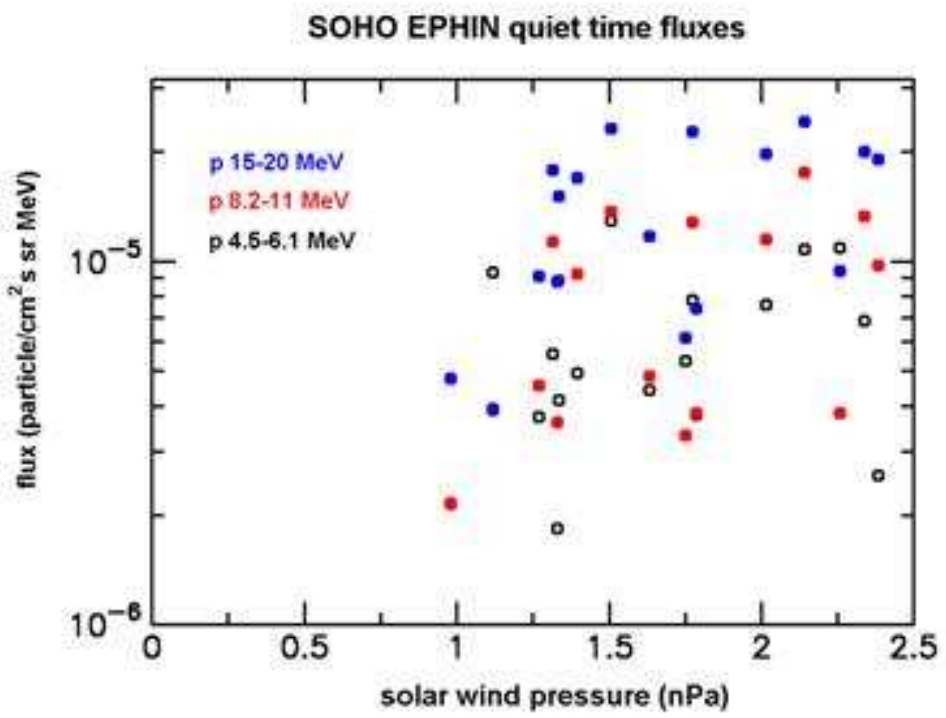

Fig. 6 Scatter plot between Solar wind pressure and quiet-time particle flux for three different energy ranges. 
interplanetary CMEs (ICMEs) and the shocks driven by them from $1 \mathrm{AU}$ to the location of Voyager 2. They showed that the ICME shock relationship is not simple as one observes at $1 \mathrm{AU}$ : successive merging and interaction of relatively small interplanetary shocks could form a well-developed strong forward shock beyond $30 \mathrm{AU}$. They showed that the strong shock (speed jump $\sim 100 \mathrm{~km} / \mathrm{s}$ ) of 2001 October 16 was due to the merging of a series of shocks observed at Earth in April 2001. They also examined the characteristics of the termination shock in detail, making use of multiple shock crossing of Voyager 2 in August 2007. For two crossing events, the flow was found to be still supersonic with respect to the thermal ions downstream of the termination shock, probably due to the fact that most of the solar wind energy is transferred to pickup ions. They concluded that the solar wind in the outer heliosphere is fundamentally different from that in the inner heliosphere, since the influence of the local interstellar source becomes significant.

\subsection{Solar Wind Structure and Cosmic-ray Modulation}

One of the implications of the solar wind and the magnetic field it carries is its impact on the galactic cosmic rays that enter the heliosphere. Ferreira (2010) described the current status of numerical model computations that compare well with spacecraft observations at various energies. The basis of these calculations is the transport equation, which is solved using realistic transport parameters, model of the heliosphere (including the asymmetry caused by the relative motion between the local interstellar medium and the Sun), and the heliospheric magnetic field. Timedependent modulation was also discussed to show that drift effects together with propagating diffusion barriers are responsible for modulation over a solar cycle.

\section{Concluding Remarks}

The summary provided in this paper gives only a birds eye view of a limited number of heliophysical processes. However, the topics mentioned are of current interest both from physics and practical points of view. The origin of solar wind and CMEs continue to occupy the forefront of solar-terrestrial research because they have been identified as the primary sources of space weather. The past decade has seen an enormous growth in research focusing on space weather not only because of its scientific research, but also because of the increased dependency of the humans on space based technology, which is vulnerable to space weather. With the recent launch of the Solar Dynamics Observatory (SDO), there will be more focus on the source of mass emission using the unprecedented SDO imagery coupled with what is already available from ground and spacebased assets. While the past decades have seen man made instruments making in situ measurements all the way to the edge of 
the heliospace, the current decade will see in situ measurements made from close to the Sun where the mass emission begins using the Solar Probe Plus mission.

Acknowledgements The author thanks Drs. K. Kecskeméty, B. Vršnak and B. J. Thompson for their help in convening the IAGA session. He also thanks Dr. P. Mäkelä for proof reading the manuscript.

\section{References}

Ahmad QR, Allen RC, Andersen TC, Anglin JD, Bühler G, Barton JC, Beier EW, Bercovitch M, Bigu J, Biller S, Black RA, Blevis I, Boardman RJ, Boger J, Bonvin E, Boulay MG, Bowler MG, Bowles TJ, Brice SJ, Browne MC, Bullard TV, Burritt TH, Cameron K, Cameron J, Chan YD, Chen M, Chen HH, Chen X, Chon MC, Cleveland BT, Clifford ET, Cowan JH, Cowen DF, Cox GA, Dai Y, Dai X, Dalnoki-Veress F, Davidson WF, Doe PJ, Doucas G, Dragowsky MR, Duba CA, Duncan FA, Dunmore J, Earle ED, Elliott SR, Evans HC, Ewan GT, Farine J, Fergani H, Ferraris AP, Ford RJ, Fowler MM, Frame K, Frank ED, Frati W, Germani JV, Gil S, Goldschmidt A, Grant DR, Hahn RL, Hallin AL, Hallman ED, Hamer A, Hamian AA, Haq RU, Hargrove CK, Harvey PJ, Hazama R, Heaton R, Heeger KM, Heintzelman WJ, Heise J, Helmer RL, Hepburn JD, Heron H, Hewett J, Hime A, Howe M, Hykawy JG, Isaac MC, Jagam P, Jelley NA, Jillings C, Jonkmans G, Karn J, Keener PT, Kirch K, Klein JR, Knox AB, Komar RJ, Kouzes R, Kutter T, Kyba CC, Law J, Lawson IT, Lay M, Lee HW, Lesko KT, Leslie JR, Levine I, Locke W, Lowry MM, Luoma S, Lyon J, Majerus S, Mak HB, Marino AD, McCauley N, McDonald AB, McDonald DS, McFarlane K, McGregor G, McLatchie W, Drees RM, Mes H, Mifflin C, Miller GG, Milton G, Moffat BA, Moorhead M, Nally CW, Neubauer MS, Newcomer FM, Ng HS, Noble AJ, Norman EB, Novikov VM, O'Neill M, Okada CE, Ollerhead RW, Omori M, Orrell JL, Oser SM, Poon AW, Radcliffe TJ, Roberge A, Robertson BC, Robertson RG, Rowley JK, Rusu VL, Saettler E, Schaffer KK, Schuelke A, Schwendener MH, Seifert H, Shatkay M, Simpson JJ, Sinclair D, Skensved P, Smith AR, Smith MW, Starinsky N, Steiger TD, Stokstad RG, Storey RS, Sur B, Tafirout R, Tagg N, Tanner NW, Taplin RK, Thorman M, Thornewell P, Trent PT, Tserkovnyak YI, van Berg R, van de Water RG, Virtue CJ, Waltham CE, Wang J, Wark DL, West N, Wilhelmy JB, Wilkerson JF, Wilson J, Wittich P, Wouters JM, Yeh M (2001) Measurement of the Rate of $v_{e}+d \longrightarrow p+p+e^{-}$Interactions Produced by ${ }^{8} \mathrm{~B}$ Solar Neutrinos at the Sudbury Neutrino Observatory. Phys Rev Lett 87(7):071,301, DOI 10.1103/PhysRevLett.87.071301, arXiv:nucl-ex/0106015

Bavassano B (2010) MHD Turbulence in the Heliosphere. In: Hulqvist B (ed) IAGA Special Sopron Book Series, Springer, Dordrecht, The Netherlands, vol This volume, $\mathrm{p} 0$

Brajša R, Wöhl H, Hanslmeier A, Verbanac G, Ruždjak D, Cliver E, Svalgaard L, Roth M (2009) On solar cycle predictions and reconstructions. Astron Astrop 496:855-861, DOI 10.1051/0004-6361:200810862

Burlaga L, Sittler E, Mariani F, Schwenn R (1981) Magnetic loop behind an interplanetary shock - Voyager, Helios, and IMP 8 observations. J Geophys Res 86:6673-6684, DOI 10.1029/JA086iA08p06673

Chandra R, Pariat E, Schmieder B, Mandrini CH, Uddin W (2010) How Can a Negative Magnetic Helicity Active Region Generate a Positive Helicity Magnetic Cloud? Sol Phys 261:127-148, DOI 10.1007/s11207-009-9470-2, 0910.0968

Charbonneau P (2005) Dynamo Models of the Solar Cycle. Living Reviews in Solar Physics 2:2

Chen J, Howard RA, Brueckner GE, Santoro R, Krall J, Paswaters SE, St Cyr OC, Schwenn R, Lamy P, Simnett GM (1997) Evidence of an Erupting Magnetic Flux Rope: LASCO Coronal Mass Ejection of 1997 April 13. Astrophys J Lett 490:L191+, DOI 10.1086/311029 
Choudhuri AR, Chatterjee P, Nandy D (2004) Helicity of Solar Active Regions from a Dynamo Model. Astrophys J Lett 615:L57-L60, DOI 10.1086/426054

Couvidat S (2010) Solar Oscillations: Current Trends. In: Hulqvist B (ed) IAGA Special Sopron Book Series, Springer, Dordrecht, The Netherlands, vol This volume, $\mathrm{p} 0$

Davila J, Gopalswamy N, Thompson B, Bogdan T, Hapgood M (2010) International Space Weather Iniative (ISWI). In: Hulqvist B (ed) IAGA Special Sopron Book Series, Springer, Dordrecht, The Netherlands, vol This volume, $\mathrm{p} 0$

Demetrescu C, Dobrica V, Maris G (2009) Signature of Hale and Gleissberg Solar Cycles in the Heliosphere-Magnetosphere Environment, IAGA abstract \#403-FRI-O1045-0292

Eddy JA (1976) The Maunder Minimum. Science 192:1189-1202

Ferreira S (2010) Cosmic Rays and Solar Activity. In: Hulqvist B (ed) IAGA Special Sopron Book Series, Springer, Dordrecht, The Netherlands, vol This volume, p 0

Gary SP (2009) Short-wavelength Solar Wind Turbulence: the Role of Whistler Fluctuations, IAGA abstract \#403-SAT-O0915-1144

Georgieva K (2009) Solar Dynamo, Interplanetary Magnetic Field, and Terrestrial Climate, IAGA abstract \#403-FRI-O1030-0324

Gopalswamy N, Mäkelä P, Xie H, Yashiro S (2009a) White-light Observations of Radio-quiet and Radio-loud Interplanetary Shocks, IAGA abstract \#403-FRI-O1415-1318

Gopalswamy N, Thompson WT, Davila JM, Kaiser ML, Yashiro S, Mäkelä P, Michalek G, Bougeret J, Howard RA (2009b) Relation Between Type II Bursts and CMEs Inferred from STEREO Observations. Sol Phys 259:227-254, DOI 10.1007/s11207-009-9382-1

Kecskeméty K, Daibog E, Zeldovich M, Logachev Y (2009) Solar Wind and MeV Energy Protons, IAGA abstract \#403-SAT-O1530-0530

Kikuchi T, Shinbori A, Tsuji Y, Hashimoto KK, Watari SI, Araki T, Abdu AM, Su SY (2010) Instantaneous Development of Global Ionospheric Currents During Geomagnetic Sudden Commencements. In: Hulqvist B (ed) IAGA Special Sopron Book Series, Springer, Dordrecht, The Netherlands, vol This volume, p 0

Kudela K, Lazutin L (2010) Solar Influence on the Magnetosphere: Information by Cosmic Rays. In: Hulqvist B (ed) IAGA Special Sopron Book Series, Springer, Dordrecht, The Netherlands, vol This volume, $\mathrm{p} 0$

Lavraud B, Gosling JT, Rouillard AP, Fedorov A, Opitz A, Sauvaud J, Foullon C, Dandouras I, Génot V, Jacquey C, Louarn P, Mazelle C, Penou E, Phan TD, Larson DE, Luhmann JG, Schroeder P, Skoug RM, Steinberg JT, Russell CT (2009) Observation of a Complex Solar Wind Reconnection Exhaust from Spacecraft Separated by over $1800 \mathrm{R}_{E}$. Sol Phys 256:379_ 392, DOI 10.1007/s11207-009-9341-x

Leighton MM, Brophy JA (1961) Illinoian Glaciation in Illinois. J Geol 69:1-31, DOI $10.1086 / 626713$

Lie-Svendsen O (2010) The Solar Wind. In: Hulqvist B (ed) IAGA Special Sopron Book Series, Springer, Dordrecht, The Netherlands, vol This volume, $\mathrm{p} 0$

Linton M (2010) Flux Ropes in the Heliosphere. In: Hulqvist B (ed) IAGA Special Sopron Book Series, Springer, Dordrecht, The Netherlands, vol This volume, $\mathrm{p} 0$

Longcope DW, Fisher GH, Pevtsov AA (1998) Flux-Tube Twist Resulting from Helical Turbulence: The Sigma-Effect. Astrophys J 507:417-432, DOI 10.1086/306312

Malandraki OE, Marsden RG, Lario D, Tranquille C, Heber B, Mewaldt RA, Cohen CMS, Lanzerotti LJ, Forsyth RB, Elliott HA, Geranios A (2009) Solar Energetic Particle Observations and Propagation in the 3-D Heliosphere in December 2006, IAGA abstract \#403-SAT-O1445-0335

Messerotti M (2010) Radio Emission Processes as Tracers of Heliospheric Weather. In: Hulqvist B (ed) IAGA Special Sopron Book Series, Springer, Dordrecht, The Netherlands, vol This volume, $\mathrm{p} 0$

Nanan B, Shiokawa K, Otsuka Y, Kikuchi T (2010) Solar Influence on Earth's Ionosphere. In: Hulqvist B (ed) IAGA Special Sopron Book Series, Springer, Dordrecht, The Netherlands, vol This volume, $\mathrm{p} 0$

Opitz A, Karrer R, Wurz P, Galvin AB, Bochsler P, Blush LM, Daoudi H, Ellis L, Farrugia CJ, Giammanco C, Kistler LM, Klecker B, Kucharek H, Lee MA, Möbius E, Popecki M, Sigrist M, 
Simunac K, Singer K, Thompson B, Wimmer-Schweingruber RF (2009a) Temporal Evolution of the Solar Wind Bulk Velocity at Solar Minimum by Correlating the STEREO A and B PLASTIC Measurements. Sol Phys 256:365-377, DOI 10.1007/s11207-008-9304-7

Opitz A, Sauvaud JA, Fedorov A, Wurz P, Luhmann J, Lavraud B, et al (2009b) Sol. Phys.: Submitted

Parker EN (1958) Dynamics of the Interplanetary Gas and Magnetic Fields. Astrophys J 128:664, DOI $10.1086 / 146579$

Petrovay K (2010) Forecasts for the Next Solar Activity Cycle and Dynamo Theory. In: Hulqvist B (ed) IAGA Special Sopron Book Series, Springer, Dordrecht, The Netherlands, vol This volume, $\mathrm{p} 0$

Poletto M (2010) Current Sheets in the Solar Atmosphere. In: Hulqvist B (ed) IAGA Special Sopron Book Series, Springer, Dordrecht, The Netherlands, vol This volume, p 0

Sung S, Marubashi K, Cho K, Kim Y, Kim K, Chae J, Moon Y, Kim I (2009) A Comparison of the Initial Speed of Coronal Mass Ejections with the Magnetic Flux and Magnetic Helicity of Magnetic Clouds. Astrophys J 699:298-304, DOI 10.1088/0004-637X/699/1/298

Terasawa T (2010) Shocks in the Heliosphere. In: Hulqvist B (ed) IAGA Special Sopron Book Series, Springer, Dordrecht, The Netherlands, vol This volume, $\mathrm{p} 0$

Török T, Kliem B, Berger MA, Démoulin P, van Driel-Gesztelyi L (2009) Rotation of Erupting Magnetic Flux Ropes in Coronal Mass Ejections, IAGA abstract \#403-FRI-O1145-0274

Valtonen E (2010) Solar Energetic Particles. In: Hulqvist B (ed) IAGA Special Sopron Book Series, Springer, Dordrecht, The Netherlands, vol This volume, $\mathrm{p} 0$

Verbanac G, Brajsa R, Cliver E, Svalgaard L, Woehl H, Roth M, Hanslmeier A (2009) An Improved Prediction for the Solar Cycle 24, IAGA abstract \#403-FRI-O1100-1025

Veronig AM (2010) Large-scale Coronal Waves Associated with Flares and CMEs. In: Hulqvist B (ed) IAGA Special Sopron Book Series, Springer, Dordrecht, The Netherlands, vol This volume, $\mathrm{p} 0$

Vörös Z, Leubner M (2009) Magnetic Turbulence is not Omnipresent in the Solar Wind, IAGA abstract \#403-SAT-O0900-0793 